\title{
Emplazando la utopía. Reinvenciones del sujeto, la comunidad y el espacio habitado en Chile
}

\section{Locating utopia. Reinventions of the individual, the community and the inhabited space in Chile}

\author{
Rodolfo E. Mardones 1 y y Hugo M. Zunino² (1)
}

\begin{abstract}
RESUMEN
Las comunidades intencionales utópicas (CIU) las entendemos como personas que se reúnen en torno a ideales para experimentar modos de convivencia que ofrecen órdenes socioespaciales alternativos contrastantes con el mundo moderno. La literatura destaca la intensificación del fenómeno en todo el mundo durante las últimas décadas. Este trabajo representa un esfuerzo por entender la diversidad de expresiones que adquieren las CIU en Chile. A partir de la revisión de documentos, entrevistas y observación participante en tres comunidades emplazadas en Chile, este trabajo ofrece una lectura de las prácticas y discursos que sostienen estos agrupamientos. Para capturar la diversidad existente, sugerimos distinguir entre comunidades religiosas, espirituales y pragmáticas. Además, argumentamos que estos espacios dinámicos están atravesados por contradicciones cuando se articulan, a partir de la capacidad reflexiva del sujeto, como un contrapunto creativo a la dinámica social capitalista.
\end{abstract}

Palabras clave: sujeto, utopía, comunidad, lugar.

\begin{abstract}
Utopian intentional communities are here understood as persons gathered around ideals to experience with models of coexistence and that suggest spatial and social orderings that contrast to the modern world. Recent contributions highlight the upsurge of the phenomena during the last decades all over the world. However, we lack studies that understand the diversity that exist in these settlements. Based on documentary analysis, interviews, and participant observation in three communities located in Chile, this work offers a lecture of the practices and discourses that sustain these settlements. To capture the existing diversity, we suggest distinguishing between religious, spiritual, and pragmatic communities. Further, we argue that these dynamic spaces are crossed by contradictions when they articulate, from the reflexive capacity of the subject, as a creative counterpoint to the capitalist social dynamics.
\end{abstract}

Keywords: Self, utopia, community, place. 


\title{
RESUMO
}

\begin{abstract}
Entendemos as Comunidades Utópicas Intencionais (IUC) como pessoas que se reúnem em torno de ideais para experimentar modos de vida que oferecem ordens sócio-espaciais alternativas em contraste com o mundo moderno. A literatura destaca a intensificação do fenômeno em todo o mundo durante as últimas décadas. Este trabalho representa um esforço para compreender a diversidade de expressões adquiridas pelo CIU no Chile. Com base na revisão de documentos, entrevistas e observação dos participantes em três comunidades localizadas no Chile, este trabalho oferece uma leitura das práticas e discursos que sustentam estes agrupamentos. Para capturar a diversidade existente, sugerimos que se faça a distinção entre comunidades religiosas, espirituais e pragmáticas. Além disso, argumentamos que estes espaços dinâmicos são atravessados por contradições quando são articulados, baseados na capacidade reflexiva do sujeito, como um contraponto criativo à dinâmica social capitalista.
\end{abstract}

Palavras-chave: sujeito, utopia, comunidade, lugar.

En las últimas décadas se ha advertido que la sociedad contemporánea enfrenta una crisis que se expresa en los diversos impactos sobre la vida humana regida por el capital, mientras persiste la fantasía de la posibilidad de un progreso infinito en un planeta con recursos limitados (Liodakis, 2018; Stubblefield, 2018). Sin embargo, también evidenciamos intentos colectivos por responder a esta situación (Mardones, 2015). En efecto, la literatura evidencia la emergencia de iniciativas comunitarias que grafican respuestas a los síntomas de una crisis, que más allá de la dimensión coyuntural, sugieren órdenes sociales y espaciales distintos a aquellos que son convencionales en nuestra sociedad (Huiliñir y Zunino, 2017; Zunino y Huiliñir, 2019). En este trabajo nos referimos a estas iniciativas que buscan responder a la crisis y transformar a la sociedad como Comunidades Intencionales Utópicas (CIU). Las CIU se pueden definir en términos generales cómo un grupo de personas que se reúnen en torno a ideales y que desarrollan modos de convivencia que les permiten concretar una forma de vida juntos orientada por sus convicciones (Christian, 2009). En los últimos años las CIU han sido estudiadas como espacios colectivos basados en propósitos ecológicos y prácticas heterogéneas, lo cual muestra una amplia diversidad. Sin embargo, la literatura es confusa respecto a cómo definir y establecer los límites de estos proyectos, ya que se carece de distinciones para reconocer la dimensión utópica como un elemento constitutivo de este tipo de comunidades. En este caso, entendemos que, a diferencia de las comprensiones clásicas sobre la utopía, como un espejismo irrealizable, esta será comprendida como un ejercicio imaginativo de otros modos de vida que moviliza a los sujetos a su concreción con un esfuerzo por reconceptualizar la modernidad (Saracho, Herrera y González, 2016). Así, nos interesa conocer las CIU que se reúnen en torno a ideales ecológicos y que se emplazan en un territorio para experimentar modelos de convivencia que manifiestan un quiebre con el mundo moderno (Escorihuela, 2008). De esta forma, articulan discursos contrahegemónicos, poniendo un claro énfasis en la concreción de cambios individuales, comunitarios y la realización de rupturas espaciales (Huiliñir y Zunino, 2017).

En la actualidad, la presencia de CIU es extensa a escala planetaria (Salamanca, 2015). Se habla de más de 100.000 personas involucradas en distintas iniciativas (Fellowship for Intentional Community [FIC], 2016) y de 15.000 proyectos utópicos de carácter ecológico activos alrededor del mundo (Global Ecovillage Network [GEN], 2018). La amplia presencia de iniciativas a nivel mundial nos ha parecido relevante para nuestra investigación, debido a que no solo observamos una expansión alrededor del mundo, sino que también una amplia diversidad, lo cual muestra su vigencia para tensionar el proyecto de la modernidad fundado en una racionalidad que nos separa 
del entorno. Más aun, nuestro caso de estudio, Chile, reúne condiciones únicas como laboratorio al estar dotado de lugares singulares con alto valor ecológico que atraen proyectos utópicos y la vigencia de un proyecto de desarrollo neoliberal en su versión más ortodoxa en el mundo (Borzutzky, 2005; Clark, 2018). Debido a la penetración del capital y la desregulación por parte del Estado en una serie de materias de vital importancia para la conservación de la vida y la convivencia comunitaria, emergen una serie de utopías, que se dirigen por un impulso propositivo autónomo, como medidas paliativas ante la perversidad del capital sobre la vida cotidiana (Escobar, 2003).

En la literatura hemos evidenciado la presencia de comunidades en algunas regiones de Chile. Estos estudios dirigen la atención hacia sus prácticas de sustentabilidad y su potencial para el cambio social (Pereira, 2013). Y han sido entendidas como el resultado de una práctica migratoria motivada por la búsqueda de un estilo de vida distinto al urbano, en contraste a la elección utópica y emotiva de lugares con alto valor paisajístico como la Araucanía andina (Huiliñir y Zunino, 2017). En similar línea, otros estudios han focalizado su atención en el valle del Elqui, en el norte del país, inserto en un incipiente mercado de la espiritualidad y la religión (Bahamondes et al., 2017). La emergencia de proyectos comunitarios que intentan responder a las condiciones impuestas por el capital también ha sido abordada desde la perspectiva de los movimientos sociales (Cancino, 2018). En esta investigación trabajamos las características de las CIU en Chile a partir de los sujetos que las construyen, poniendo el énfasis en los gestores de estas iniciativas utópicas. De esta manera nuestra intención, a diferencia de estudios previos, se centra en sus prácticas discursivas y significados que anuncian que otros mundos son posibles. Las tensiones emanadas desde estas formas de vida comunitaria hacia la sociedad actual, y la escasa visibilidad de estos proyectos en el sur del continente americano, nos llevan a preguntarnos: ¿Qué características tienen estos espacios comunitarios utópicos en Chile? y ¿Quiénes son los sujetos que participan en la construcción de estas iniciativas utópicas? Para responder nuestras preguntas, investigamos orientados por una perspectiva cualitativa. En una primera fase indagamos la situación de 49 comunidades utópicas emplazadas en Chile. En una segunda fase, focalizamos nuestro trabajo de campo en tres casos de estudio con el propósito de profundizar en la diversidad de sus propuestas.

Este trabajo se organiza en cinco secciones: en un primer momento presentamos sintéticamente las concepciones de sujeto moderno y la emergencia de utopías comunitarias. Posteriormente, nos detenemos en antecedentes sobre CIU y las características de los sujetos involucrados. Luego exponemos nuestras estrategias metodológicas desplegadas. En los resultados reportamos los principales hallazgos sobre las CIU en Chile y nos detenemos en su propuesta de reinvención espacial, comunitaria y de los sujetos involucrados. Concluimos exponiendo la distribución de estas comunidades en el territorio, los conocimientos que sustentan sus formas ideales de vida en común y el rol del sujeto en el contexto social actual.

\section{Sujeto moderno y utopía comunitaria}

Chile es heredero y parte constituyente del proyecto moderno europeo, insertándose en un doble movimiento: la colonialidad (Lander, 2000) y la racionalidad neoliberal (Castro-Gómez, 2015). El proyecto ontológico moderno levantó como principal valor la razón universal por sobre concepciones ancestrales, religiosas o espirituales (Ferreira, 2013). Una de las iniciativas centra- 
les fue el cambio en la concepción de sujeto, en donde la figura del individuo consciente de sus propios intereses, sentimientos y metas desplaza los centros teológicos, políticos y epistemológicos, que se habían usado como referencias privilegiadas de sentido. El individuo aparece como el correlato de unas instancias internas y de otras externas que configuran las representaciones, imágenes y los patrones que proceden del entorno social (Beriain, 2011). Esta comprensión establece consecuencias que se traducen en múltiples dualismos como, por ejemplo, razón-creencia, mente-cuerpo, libertad-dependencia, las cuales cristalizan una distinción binaria en todas las dimensiones de la vida social moderna (Lander, 2000). En este contexto, la constitución del sujeto moderno se caracteriza por el desarrollo de una interioridad psíquica y la emergencia de su capacidad de expresión. Desde esta perspectiva el yo se entiende como constituido por una serie de fuentes morales que lo llevan a un ideal con capacidad de expresarse auténticamente (Taylor, 2006). En particular, tiene la posibilidad de escoger, decidir, crear y aspirar a ser autor de una identidad individual. Esta individuación caracteriza al sujeto moderno, ya que su biografía no solo es socialmente prescrita, sino que puede ser racionalmente constituida (Beriain, 2011).

En este sentido, aumentan los constreñimientos que configuran la propia biografía y saturan el yo por una pluralidad de voces (Gergen, 2006). Por ejemplo, los individuos libres, gracias a sus posibilidades racionales, se convierten en dependientes del mercado, del trabajo, la formación escolar, el consumo o las regulaciones del Estado (Beriain, 2011). A pesar de estas directrices institucionales, el sujeto se dirige a la actividad y con ello su movilidad se convierte en la condición de posibilidad del capitalismo, el cual aparece no solo como productor de mercancías, sino que también de subjetividades (Castro-Gómez, 2009). Es en esta indeterminación existente entre la liberación y la dependencia, donde se sitúa la ambivalencia moderna (Bauman, 2005). Llevando a la concepción de un sujeto inacabado, movilizado por el deseo y constituyente de un proyecto de autorrealización individual que lo configura como un empresario de sí mismo, el cual puede desempeñar estrategias en torno a la creatividad y el empoderamiento, movilizando distintos recursos orientados por la racionalidad neoliberal dirigidos como fin último al proyecto de uno mismo (Bröckling, 2015). La búsqueda del sujeto va acompañada con la expresión de desilusión y desencanto, debido a que esta dinámica lo obliga al rendimiento, a responsabilizarse de su gestión interna y de su desempeño en la sociedad, cuestión que provocará su frustración, angustia y, en el peor de los casos, depresión (Vásquez, 2014; Han, 2014).

En el mismo entramado donde el sujeto se constituye como racional y se disputa entre una interioridad psíquica radical o una exterioridad neoliberal que se presenta como natural, observamos la emergencia de utopías que tensionan a este sujeto moderno. Este impulso por trascender los márgenes de la modernidad se caracteriza por un pensamiento que se opone a la instrumentalización de la razón, abierto a la multiplicidad de juegos de lenguaje, lo cual va en paralelo a una crisis sobre la concepción de la realidad, en donde el esquema sujeto-objeto es tensionado en términos ontológicos. Algunos autores han conceptualizado estos movimientos como "utopías postmodernas", haciendo referencia al carácter diferente que presentan estos proyectos en relación con la modernidad (González, 2010).

Sin embargo, se advierte que el ejercicio de imaginar, relativizar y rediseñar las racionalidades de la vida en común puede reconocer antecedentes antiguos (Nogué, 1988). Por ejemplo, en Platón, en su obra La Republica como una propuesta de ciudad ideal, lo cual es retomado de forma más explícita por Tomás Moro (1952) en su libro ampliamente divulgado, Utopía, el cual 
expone ideales políticos del mundo clásico y del cristianismo como una forma alternativa de vida en una lejana isla. También podemos referir antecedentes que, con algunos matices, surgieron a partir del siglo XIX como respuesta al malestar provocado por la industrialización, es el caso del movimiento garden city, propuesto por Ebenezer Howard, en donde la relación humano y naturaleza toma un lugar central en la planificación urbana. Desde una posición más radical, Henry Thoreau propone en su libro Walden (1949), la opción de un individuo en ruptura con la sociedad y dispuesto a establecer una relación diferente con la naturaleza. Por otro lado, y con un énfasis comunitario, se encuentra la propuesta del francés Charles Fourier, quien diseña una forma de vida alternativa inspirada en la propuesta del socialismo utópico (Fourier, 1989). En estos casos constatamos argumentos que posteriormente serán retomados por comunidades utópicas más contemporáneas como los Kibbutz en Israel (Achouch y Morvan, 2012). Por otro lado, el movimiento hippie en los años sesenta en Estados Unidos, y las iniciativas comunitarias inspiradas en las propuestas de la novela "Walden two" (Skinner, 1968), en donde, haciendo un gesto hipertextual con la propuesta de Thoreau, el psicólogo conductista proponía una utopía psicológica y conductual para la vida en común a pequeña escala (Ardila, 2004).

Esta retrospección es importante, ya que muestra antecedentes sobre la posibilidad de responder a lo instituido y proponer la construcción de un nuevo ser humano desde comprensiones utópicas. Sin embargo, también nos muestra un contraste respecto a su anecdótica presencia en el pasado y su intensificación en la sociedad contemporánea, lo cual nos lleva a entenderlo como un impulso propio del ser humano en el contexto de la modernidad y el capitalismo.

\section{Comunidades intencionales utópicas: reinvención del sujeto y del espacio habitado.}

Un elemento central para la definición de una CIU es la intencionalidad, en este sentido, por ejemplo, en América anglosajona se ha propuesto un directorio para distinguir las comunidades entre ecoaldeas, coviviendas, comunas, cooperativas, comunidades judías y católicas (FIC, 2016). Esto devela una gran diversidad y, al mismo tiempo, confusión en su comprensión; ya que evidencia matices y desencuentros en su definición (Choi, 2008). En este contexto, nos parece que, más allá de destacar exclusivamente la intencionalidad para establecer una vida en común, es relevante distinguir elementos dirigidos a la interpelación de la modernidad mediante un proyecto utópico que se orienta a la experimentación de formas de vida que se alejan de los ideales promovidos por el capitalismo (Saracho, Herrera y González, 2016). Al respecto, la literatura ha iniciado una interesante discusión que refiere que el utopismo de estos grupos es profundamente imperfecto y práctico, enraizado en las preocupaciones y limitaciones materiales del presente (Figueroa y López, 2014; Sargisson, 2012; 2007). La vida cotidiana de los miembros son experimentos en curso, y esta experimentación ocurre en muchos niveles, sustentando las estructuras físicas y sociales en las que coexisten la naturaleza de sus relaciones, y otros asuntos más amplios como sus actividades, expectativas económicas, éticas y espirituales (Sargisson, 2009). En esta gran diversidad, reconocemos que, en general, los miembros comparten una situación de insatisfacción respecto a lo que les ofrece la vida modernizada y empatizan con ideas ecologistas que informan su estilo de vida (Van Kerkhoff y Lebel, 2006). Sin embargo, en la literatura identificamos tendencias, que, a nuestro juicio permiten hacer algunas diferenciaciones entre las CIU. En este sentido, de acuerdo con Mathews (2006), diferenciamos aproximaciones que focalizan en lo reli- 
gioso, espiritual o pragmático. Evidenciamos la comprensión de lo religioso como un sistema de creencias metafísicas o enseñanzas prescriptivas a las que han llegado de forma extra-racional o por la revelación, las cuales son grabadas en textos sagrados que luego son mediados por autoridades religiosas que forman parte de una orgánica institucional. La espiritualidad, en cambio, nos acerca a la experiencia de una realidad interior trascendente que va más allá de lo físico-visible, la cual no es anticipada por reglas ni mediada por una autoridad como en el caso de la religión. Finalmente, lo pragmático hace referencia a posiciones en donde dominan las relaciones materiales por sobre concepciones místicas y en donde se privilegia la razón instrumental y científica para intervenir en el mundo con fines humanos.

De acuerdo con la distinción realizada a partir de la literatura, las CIU de orientación religiosa desarrollan una vida que se grafica en la práctica de ritos y ceremonias orientadas por su doctrina. Exteriorizan su espiritualidad mediante la veneración a dioses, mediada por escrituras sagradas. La literatura ha destacado el rol de comunidades judías y los kibutz (Achouch y Morvan, 2012). También se ha reportado la existencia de comunidades cristianas en el ámbito de la covivienda (FIC, 2016). Desde un punto de vista más crítico con el materialismo de la modernidad occidental, se ha relevado el caso de las comunidades Hare Krishnas como un movimiento religioso y ecológico que articula una red de templos y ecoaldeas en diferentes países (Eco-Yoga Villages, 2018). Los devotos de Krishna sustentan sus comunidades en una economía de grupo, un mínimo número de posesiones, una dieta vegetariana y comparten todos los espacios cotidianos (Chaves, 2018).

Para el caso de las CIU de orientación espiritual, inferimos que estas comunidades se orientan a la trascendencia del sujeto y buscan una relación armónica con la naturaleza. Esta espiritualidad, se entiende como experiencia interna del sujeto $y$, las comunidades, se proponen como espacios de transformación personal y vida espiritual (Litfin, 2014), incluso se atribuye a sus prácticas el potencial de ensayo para una humanidad superior (Quiroga, 2014). El cambio personal depende de los valores asumidos por la comunidad (Christian, 2009) y el objetivo de fondo es tomar una posición crítica frente al sujeto moderno, por la vía de la comunión de individuos conscientes de sí mismos y de la historia personal que los constituye (Escorihuela, 2008). En este contexto, el rol de los sujetos es central, ya que se movilizan en torno a ideales y los concretan junto a otros, manteniendo formas de relación basadas en la cooperación (Kirby, 2003). En este sentido, se han relevado los propósitos y actividades que se realizan cotidianamente para concretar la trasformación personal como medio para un cambio en la sociedad (Silvestri, 2015). Para esto, experimentan con prácticas espirituales diversas con el objetivo de expandir su conciencia, recurriendo a orientaciones como, por ejemplo: neochamanismo, budismo, krishnaísmo y espiritualidad new age (Berger, 2006).

Por otro lado, entendemos que las CIU de orientación pragmática focalizan en la transición ecológica. Reconocemos su pragmatismo en la implementación de acciones dirigidas a la solución de necesidades de la vida cotidiana, a través del desarrollo de tecnologías e innovaciones. Por ejemplo, en la agricultura y producción agroecológica (Eksvärd y Rydberg, 2010), en el manejo del suelo (Kim et al., 2006) y el uso del agua (Hood et al., 2010). También en la producción de energías limpias (Ho, Hashim y Lim, 2014). Así como el diseño y construcción de viviendas sustentables (Lockyer, 2008). Se evidencia la presencia de saberes expertos que permiten la concreción de iniciativas de autosuficiencia (Sullivan, 2016), los cuales se matizan con saberes tradicionales y la permacultura como estrategia agroecológica viable para el diseño social (Walker, 1996). Se 
constata la propuesta de una relación ecosistémica con la naturaleza orientada al desarrollo humano, calidad de vida y estrategias de bienestar (Hall, 2015), articulando su trabajo en una amplia red de ecoaldeas (GEN, 2018).

En general, identificamos que las diferencias entre las CIU no son fijas y muestran distintas formas de organización y vinculación con la sociedad, en algunos casos las comunidades se reportan como instancia de turismo bajo la denominación de ecoturismo (Rajović y Bulatović, 2015) o prestación de servicios eco-espirituales y terapéuticos diversos para el bienestar integral u holístico de los sujetos. De esta forma, las CIU también evidencian espacios híbridos de sostenibilidad económica. En algunos casos se trata de formas de comercialización subrepticias y en otros se trata de espacios alternativos a la sociedad capitalista (Cenzatti, 2008). Es a propósito de estas articulaciones con el capital, que estas iniciativas han recibido críticas respecto al bajo impacto que pueden tener para el cambio social, incluso han sido interpeladas argumentando que podrían ser parte del mismo problema que critican (Fotopoulos, 2006). Por ejemplo, se evidencian ventajas en la adquisición de la tierra según la clase social de los participantes, o prácticas de subcontratación para llevar a cabo las tareas de mantención de la comunidad (De Matheus, 2014). Las contradicciones evidenciadas en las CIU sugieren que la utopía puede usarse como un término genérico, ante lo cual es importante señalar que estos espacios encierran peligros y amenazas, por lo que pueden configurarse como autoritarios y con formas restrictivas de gobierno, vigilancia y control (Harvey, 2003).

En este contexto, una condición de posibilidad, de las CIU, es la migración de los sujetos que las constituyen. Para el caso de Chile, esta migración utópica evidencia la intención por construir órdenes socioespaciales distintos y se determina por la movilidad desde la ciudad a espacios de alto atractivo por sus características naturales únicas (Zunino \& Hidalgo, 2010). Los procesos de formación de comunidades intencionales han sido estudiados desde la noción de migración por estilo de vida, resaltando el poder de trasformación que los migrantes acarrean y sus efectos sobre el tejido sociocultural del lugar (Zunino y Huiliñir, 2019; Erices et al., 2019). En particular, estos lugares han mostrado la capacidad de atracción de sujetos en torno a la construcción de utopías que, trasversalmente, valoran la naturaleza como espacio para la concreción individual o comunitaria de sus convicciones (Huiliñir y Zunino, 2017). Es relevante constatar la intensificación de la presencia de estas comunidades. Para el caso de Chile, una serie de estudios han abordado el fenómeno a escala local. Por ejemplo, se ha avanzado en estudios que muestran inicialmente la diversidad del fenómeno, sus prácticas, la visión de sociedad y de sí mismos (Mardones y Zunino, 2019), otros han valorado estas comunidades desde la perspectiva de los movimientos sociales en términos de su incidencia en procesos políticos más generales (Cancino, 2018). Otros reportan y describen casos de estudio: en la región de Valparaíso una ecoaldea encauzada por valores dirigidos a la transición ecológica (Lombardozzi, 2017); en el norte de país, el caso de una ecoaldea que integra distintos conocimientos para la construcción de un estilo de vida holístico (Concha, 2010). Otros estudios reportan casos localizados en los Andes de sur de Chile, los cuales se caracterizan por considerar saberes indígenas, espirituales y diversas concepciones biocéntricas, en las cuales se inserta el sujeto en búsqueda de la espiritualidad y bienestar personal. Es por ello, por lo que estas comunidades han sido nominadas bajo la noción de utopía postmoderna, ya que se caracterizan por su eclecticismo y ausencia de un relato común (Zunino y Huliñir, 2016; 2017). 
Construyendo a partir de estos casos de estudio, nuestro interés en este trabajo es ofrecer una visión general sobre el desenvolvimiento del fenómeno en Chile y conocer las características de los sujetos que sostienen a estos agrupamientos utópicos.

\section{Material y Métodos}

Como muestran estudios recientes, el fenómeno de las CIU se ha intensificado en los últimos tiempos en Chile. En este contexto, las estrategias metodológicas fueron guiadas por una perspectiva cualitativa, orientada a describir las prácticas y los significados que le entregan los participantes en su vida cotidiana (Guber, 2001; Mardones, Ulloa y Salas, 2018), la cual se concretó en dos fases: 1) revisión de documentos y visitas preliminares, 2) trabajo de observación con participación en terreno y entrevistas en profundidad.

La revisión se concretó mediante una búsqueda abierta de documentos webs, con el propósito de contextualizar la extensión del fenómeno estudiado en Chile desde elementos empíricos, además de visitas preliminares. Esto nos permitió seleccionar tres casos de estudio que graficaron la diversidad del fenómeno en el país. En esta primera fase, la selección de las comunidades fue intencionada en base a criterios definidos previamente (Perez-Luco et al, 2017): (a) autodefinición como comunidad intencional u otra nomenclatura que los identificara como grupo social constituido intencionadamente, por ejemplo, ecoaldea, comunidad ecológica, ecocentro, finca ecológica, proyecto o iniciativa autosustentable; (b) expresión de una crítica a la sociedad moderna; (c) declaración de una propuesta alternativa a la sociedad actual; (d) funcionamiento de al menos un año y (e) formación de un agrupamiento emplazado en Chile.

Considerando que las comunidades usan la web ampliamente (Pereira, 2013), se efectuó una detallada revisión de la actividad web y en redes sociales a escala nacional, incluyendo todas las páginas web de CIU que estaban en funcionamiento entre septiembre de 2017 y junio de 2018. Con estos criterios se lograron identificar 49 comunidades. La revisión documental se detuvo en el material producido por las mismas comunidades en la web (páginas web, blog, redes sociales y en algunos casos documentos físicos) y complementariamente se tomó contacto con ellas, o con informantes clave, mediante visitas, correo electrónico y/o telefónicamente. Esta estrategia también fue utilizada para resolver discrepancias y triangular la información documental pesquisada. Lo anterior, nos permitió condensar información sobre la historia de las comunidades, ubicación, forma de organización social, conocimientos que las fundamentan, participantes y actividades comunitarias. Finalmente se realizó un análisis descriptivo de esta información, agrupando los enunciados de las comunidades en temáticas comunes, con el propósito de captar sus significados.

La segunda fase del estudio se sustentó en el trabajo de terreno con participantes de CIU. Con el propósito de profundizar en los antecedentes recabados en la fase previa, los casos se eligieron mediante un muestreo guiado por la teoría y con base a las evidencias empíricas (Pérez-Luco, Lagos, Mardones y Sáez, 2017). De esta forma se seleccionaron tres comunidades en Chile, una religiosa, una espiritual y una pragmática, con la intención de profundizar en la diversidad que encierran estos espacios comunitarios. 
El trabajo de campo en cada CIU se concretó mediante la observación con participación y entrevistas en profundidad con habitantes permanentes, lo cual permitió la inmersión a la vida cotidiana durante un periodo de 15 días en promedio en cada comunidad entre octubre de 2017 a enero de 2019. El trabajo de campo consistió en la permanencia y participación de sus actividades cotidianas de mantención, cocina, talleres, ceremonias, entre otras, con el propósito de comprender los sus prácticas discursivas y producción de sentidos en lo cotidiano (Spink, 2005). Consiguiente, la interacción privilegió la experiencia directa de participación en las comunidades, dando espacio a conversaciones que permitieron clarificar las explicaciones que los participantes les dan a sus prácticas desde una perspectiva no directiva (Guber, 2001). El registro se realizó mediante notas de campo y memos de síntesis grabados al final de cada día de permanencia en la comunidad. Finalmente, se realizó un análisis de los significados de las experiencias registradas mediante la organización, síntesis y categorización temática de las notas y entrevistas con los participantes. Los hallazgos se organizaron tomando como consideración ética la confidencialidad y resguardo de la identidad de los sujetos y comunidades involucradas.

\section{Resultados}

\section{Comunidades utópicas en Chile.}

De acuerdo con la revisión de documentos, constatamos que la fundación de las primeras CIU pragmáticas y religiosas fue en la década de los 90, mientras que a partir del año 2010 se fundan la mayoría de CIU espirituales. Un asunto de relevancia para la instalación de las comunidades es el acceso y propiedad de la tierra. Los casos estudiados, tanto en la revisión documental como en nuestro trabajo de campo, muestran que la propiedad privada del espacio habitado es la forma más frecuente. Sus medios de adquisición han sido a través de la compra directa por los fundadores, compra colectiva o en otros casos el acceso ha sido por herencia. Otras opciones, más periféricas en cuanto a número de casos, es la ocupación ilegal de espacios periurbanos y el usufructo. Este último se da como consecuencia de un vínculo de amistad con el/la propietario/a y se permite la instalación de la comunidad.

Las CIU, pesquisadas en la primera fase, se ubican a lo largo de todo el país (ver figura $\mathrm{N}^{\circ} 1$ ). La distancia entre las comunidades y los centros urbanos más cercanos muestran casos que se instalan en la periferia de grandes ciudades, mientras que otras superan los $60 \mathrm{~km}$ de distancia de la ciudad más cercana. En particular, 37 de las 49 comunidades identificadas, se emplazan en la ruralidad, mientras que 12 se emplazan en espacios periurbanos. En promedio, el emplazamiento en zonas periurbanas se concentra cercanos a la capital, en el litoral central. Mientras que el emplazamiento rural se da con mayor frecuencia en la Araucanía andina. Llama la atención, en esta primera fase del estudio, que los espacios geográficos en donde se ubican las comunidades se caracterizan por su alto valor paisajístico y/o potencial para la agricultura.

De estos resultados se colige que la ciudad es un elemento principal para atraer CIU en su área de influencia, las principales zonas de concentración de estas iniciativas son La Serena (Valle del Elqui), Valparaíso-Santiago, Concepción (Bio Bio) y Temuco-Pucón. El emplazamiento en estas zonas de alto valor ecológico se relaciona con que estas comunidades muchas veces acarrean en- 
Figura $\mathrm{N}^{\circ} 1$

Ubicación de comunidades intencionales utópicas en Chile
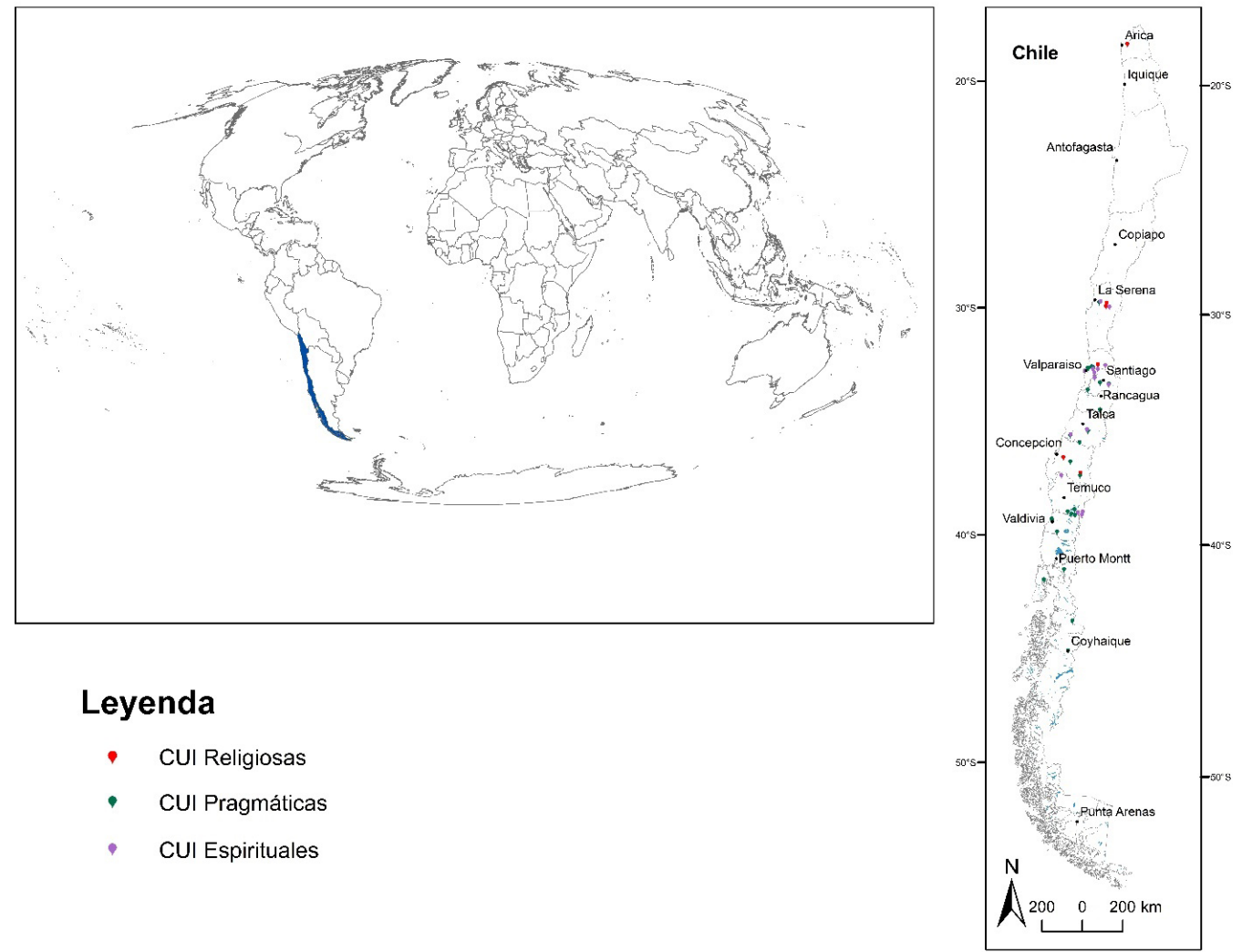

\section{Leyenda}

- CUI Religiosas

- CUI Pragmáticas

- CUI Espirituales

Fuente: Elaboración propia (por geógrafo PhD. B. Hora con datos de los autores).

tendimientos ancestrales que ven en la naturaleza la expresión de la divinidad y su presencia trae consigo entendimientos, prácticas y ritos diversos como, por ejemplo, de las culturas indígenas mesoamericanas, krishnaísmo, budismo y ecocentrismo pseudo-científico.

Los participantes de comunidades utópicas mantienen un activo vínculo con la sociedad. Sin embargo, su forma de organización y vinculación social toma distintas figuras. En efecto, la revisión documental nos permitió identificar, por ejemplo, agrupaciones que buscan influir en la sociedad a través de objetivos ecológicos o espirituales. Empresas, que buscan una producción en armonía con el medioambiente o terapias alternativas. Familias, que buscan una nueva forma de vida comunitaria fuera de la ciudad. O devotos de maestros espirituales que buscan una experiencia trascendental para el cambio social.

Lo anterior funciona como un facilitador para relacionarse e influir en la sociedad, desde las mediaciones que su forma de organización les entrega. Nuestro trabajo de campo permite argumentar que, la forma de organización de las CIU, muchas veces se trata de una decisión que permite el ensamblaje estratégico con la sociedad chilena para lograr su sustentabilidad 
económica. En lo práctico, algunas mantienen vínculos con instituciones gubernamentales, pequeñas empresas y organizaciones locales. Además, evidenciamos el vínculo que las CIU mantienen con otras comunidades con quienes comparten intereses. Sus redes no se restringen al ámbito nacional, sino que también pesquisamos importantes redes internacionales como: Global Ecovillage Network, The Ecoyoga Village, Red de Ecoaldeas Abyayala. Que, con matices, centran lo ecológico como su ámbito común de actuación (Cancino, 2018; De Matheus, 2014; Pereira; 2013).

De acuerdo con la revisión documental, un asunto definitorio de las CIU son los conocimientos ecológicos. Por un lado, observamos argumentos trascendentales orientados a la comprensión de la relación entre sujetos y naturaleza en donde se combinan conocimientos ecológicos con saberes que se centran en la capacidad reflexiva del sujeto. Si bien son variados, las CIU refieren saberes indígenas y chamanismo, meditación, yoga, constelaciones familiares, astrología, antroposofía, psicología transpersonal, entre otros. Saberes que se proponen, según sus descripciones, en una atmosfera espiritual y de aprendizaje constante, lo cual justificaría el vínculo entre el individuo y la naturaleza desde una perspectiva trascendental.

Por otro lado, evidenciamos conocimientos científicos y técnicos orientados a la transformación del lugar, los cuales entran en diálogo con saberes locales y tradicionales para delimitar la relación y los usos de la naturaleza.

Desde una comprensión distinta, evidenciamos que la religión Hare Krishna tiene gran implicación en la fundación de CIU en Chile, concretando iniciativas de fincas ecológicas y ecoaldeas, las cuales se vinculan con la naturaleza bajo una relación devocional. Por ejemplo, en el trabajo de campo constatamos una relación de servicio con la naturaleza y con los otros como propuesta de un cambio cultural amplio basado en la transformación de las conciencias individuales.

Finalmente, el trabajo de campo nos permitió dilucidar que los conocimientos, aunque son diversos y en constante permeabilidad, brindan características distintivas a las comunidades y sus participantes. Así como sus variabilidades y mixturas permiten actualizaciones en la comprensión del sujeto, las relaciones con otros y las transformaciones del lugar.

\section{Sujetos, participación y vida comunitaria.}

La participación de los sujetos y su implicación en las distintas CIU evidencia que los fundadores de las comunidades despliegan un rol central en estas, siendo los facilitadores de la cohesión del grupo y orientadores de los propósitos de la comunidad, expresado en modos de convivencia que les otorgan identidad y carácter a estos proyectos. Se trata de personas que manifiestan su preocupación por el daño al medio ambiente y las dinámicas de explotación y consumo que caracterizan nuestra sociedad.

Para profundizar en las características de los sujetos, la segunda fase de este estudio focalizó tres comunidades intencionales ecológicas con orientaciones religiosa, espiritual y pragmática (Ver cuadro $N^{\circ} 1$ ). 
Cuadro $\mathrm{N}^{\circ} 1$.

Características de las comunidades intencionales ecológicas consideradas como casos de estudio

\begin{tabular}{|l|l|l|l|}
\hline & \multicolumn{1}{|c|}{ CIU Religiosa } & \multicolumn{1}{c|}{ CIU espiritual } & \multicolumn{1}{c|}{ CIU pragmática } \\
\hline Año de fundación & \multicolumn{1}{|c|}{1997} & \multicolumn{1}{c|}{2004} & \multicolumn{1}{c|}{2007} \\
\hline Región & Coquimbo. & Coquimbo. & Bio Bio. \\
\hline $\begin{array}{l}\text { Organización } \\
\text { social }\end{array}$ & $\begin{array}{l}\text { Congregación religiosa } \\
\text { con reconocimiento le- } \\
\text { gal. }\end{array}$ & $\begin{array}{l}\text { Grupo de amigos y or- } \\
\text { ganización comunitaria } \\
\text { con reconocimiento le- } \\
\text { gal. }\end{array}$ & $\begin{array}{l}\text { Familia con iniación de } \\
\text { actividades comerciales. }\end{array}$ \\
\hline Conocimientos & $\begin{array}{l}\text { krishnaísmo, conoci- } \\
\text { miento local y ecológi- } \\
\text { co. }\end{array}$ & $\begin{array}{l}\text { Pueblos indígenas, bu- } \\
\text { dismo, constelaciones } \\
\text { familiares, ecologismo, } \\
\text { saberes locales. }\end{array}$ & $\begin{array}{l}\text { Científico (agronomía, } \\
\text { ingeniería, psicología), } \\
\text { Permacultura, saberes } \\
\text { locales. }\end{array}$ \\
\hline $\begin{array}{l}\text { Formas de } \\
\text { participación }\end{array}$ & $\begin{array}{l}\text { Devotos, voluntarios y y } \\
\text { visitantes. }\end{array}$ & $\begin{array}{l}\text { Guardianes, participan- } \\
\text { tes, voluntarios, amigos } \\
\text { y visitas. }\end{array}$ & $\begin{array}{l}\text { Familiares, amigos, vo- } \\
\text { luntarios, alumnos, visi- } \\
\text { tas. }\end{array}$ \\
\hline
\end{tabular}

Fuente: Elaboración propia

Comunidad religiosa. Las características de las personas que participan en esta comunidad intencional ecológica se caracterizan por su amabilidad y calidez en el trato. Muestran preocupación por los problemas de esta sociedad, la cual, según el encargado de la comunidad, se trata de "(...) una sociedad basada en la explotación y el desafío personal de un devoto de Krishna es relacionarse desde el servicio" (CR, febrero de 2018).

Asumir una actitud de servicio como un orientador conductual del sujeto, se grafica en su humildad, actitud paciente de aprendizaje y respeto por los demás. Sin embargo, también se pudo observar que entre los miembros de la comunidad se relacionan con una forma de cordialidad enraizada en comprensiones conservadoras, por ejemplo, en torno a las diferencias de género. El respeto hacia los otros también los lleva a respetar su organización vertical y jerárquica, la cual funciona como un templo, dónde la comunidad, fundada por un maestro espiritual, encarga la gestión de este lugar sagrado a un líder. De acuerdo con el trabajo de campo, clarificamos que la dependencia no es solo jerárquica, sino que tiene que ver con la inspiración que, alguno de los miembros, genere al mostrarse coherente y consistente en el trabajo de servir a los demás, por lo cual también existe espacio para liderazgos informales. La toma de decisiones es por consenso y comparten todos los aspectos y espacios de la vida cotidiana, comiendo, durmiendo y sirviendo juntos a Krishna.

Los participantes se muestran con optimismo sobre su impacto para la transformación de la sociedad y sobre su aporte para el cambio cultural, esto se observa en la participación de organizaciones locales para la defensa del medio ambiente. Lo cual tiene una coherencia biográfica con muchos de los participantes, quienes en su historia de vida han formado parte de instancias críticas con la sociedad, por ejemplo, participando de organizaciones estudiantiles, medioambientales, animalistas y grupos punk, entre otros.

El trabajo de campo nos permitió ver que la cocina es el espacio de articulación de aprendizaje y de servicio, en donde la comida toma un lugar protagónico como práctica identitaria de los par- 
ticipantes. Esta se vuelve el principal articulador de las relaciones consigo mismo y con los otros. Por lo tanto, las habilidades para cocinar e innovar en la cocina se plantean como una estrategia de mediación en las relaciones cotidianas, en donde las distintas preparaciones, permiten la trasmisión de una forma de ver el mundo sin sufrimiento animal.

Comunidad espiritual. Los participantes de esta comunidad intencional ecológica se definen como un grupo de amigos reunidos por su preocupación del daño al medioambiente y la falta de conciencia que caracteriza la sociedad chilena. La comunidad funciona como una instancia de encuentro para este grupo. A pasar que en la actualidad sólo una familia cumple el rol de guardianes del espacio en el lugar, la comunidad extensa abarca a todas aquellas personas que se relacionan continuamente a partir de instancias planificadas que les permiten compartir y cultivar la amistad. En este sentido, la comunidad abarca más allá de lo visible en un momento particular. En nuestro trabajo de campo accedimos a una de estas instancias y observamos que sus relaciones son horizontales y la toma de decisiones se concreta mediante el consenso, existe amabilidad y apertura a la recepción de visitas y un ejemplo de esto es permitir nuestra presencia en el lugar en uno de sus encuentros.

Refieren una preocupación por sí mismos, lo cual se manifiesta en la producción de su alimentación, las relaciones con otros, el autoconocimiento y desarrollo personal. Se muestran optimistas sobre su aporte a la sociedad y sobre su autosostenibilidad. Sin embargo, el trabajo de campo evidencia que la concreción de sus propósitos se orienta al desarrollo personal. Si bien reconocen que están caminando hacia la autosustentabilidad, también observamos que aún queda por avanzar para llegar a concretar este ideal. Por ejemplo, no logran la producción de su propio alimento ya que el terreno que ocupan es árido y no permite cubrir las necesidades cotidianas, cumpliendo más bien un rol ornamental, de conservación ambiental o de gratificación personal.

En el ámbito de su autoconocimiento y desarrollo, refieren una actitud de búsqueda y mejoramiento constante, para esto mencionan distintos saberes que se orientan a una comprensión trascendental del sujeto en armonía con la naturaleza. Los conocimientos que sustentan la búsqueda son variados: saberes terapéuticos, budismo zen, constelaciones familiares, saberes de los pueblos indígenas (Diaguita y Mapuche), psicología transpersonal y ecologismo, los cuales también agenciarían su impacto en la transformación del lugar, “(...) este es un lugar sagrado, en el sentido de que es todo un asentamiento muy antiguo de la cultura diaguita. También, es un punto donde durante más de 10 años se practicó la meditación zen. Aquí hubo un centro de meditación zen. Además, un lugar libre de pesticidas, desde hace por lo menos 30 años, igarantizado!" (CE, febrero de 2018).

El rol de los guardianes de la ecoaldea es muy importante, porque mantienen el núcleo articulador de un grupo de personas que busca, en distintas comprensiones de la realidad, opciones de crecimiento personal. Llama la atención la actitud de desprendimiento con lo material, en el trabajo de campo los mismos participantes enfatizan que el terreno no es propio, sino que había sido facilitado, gracias a una relación de confianza, y la construcción de una casa ecológica que cuenta con todas las comodidades prueba una relación de más de 10 años.

Comunidad pragmática. Las características de los participantes de esta comunidad intencional ecológica evidencian que se trata de personas con inquietud intelectual, que sustentan sus 
diagnósticos y soluciones, sobre la sociedad contemporánea, en conocimientos científico-técnicos. En efecto, su presentación pública expone que se han formado como profesionales de la ingeniería (ambiental, agronomía, industrial), planificación y agricultura. Asumiendo especialidades en postgrado en diversos ámbitos, lo cual muestra una actitud inquieta sobre la búsqueda de conocimientos y experiencias que les permita generar cambios concretos. En lo cotidiano, llama la atención la flexibilidad con la que asumen los conocimientos científicos, mostrando apertura a considerar saberes locales, en un juego creativo para buscar soluciones a los problemas ambientales de nuestra sociedad desde un punto de vista muy pragmático, para, según sus palabras, "crear un modelo que sea viable y replicable, que nos posibilite hacer lo que amamos y que ayude a otros a encontrar la inspiración para hacer lo mismo" (CP, enero de 2018).

Los/as participantes desarrollan una serie de prácticas que integran la producción orgánica de alimentos, la venta de cursos y asesorías de proyectos sustentables. En efecto, observamos que estas prácticas no solo se sostienen en motivaciones utópicas, sino que también aseguran un ingreso económico para la comunidad. Respecto de lo anterior, nuestro trabajo de campo nos permitió verificar una autoevaluación muy positiva respecto del aporte que, como comunidad, pretenden hacer al cambio social. Mostrando optimismo y apertura para compartir y mostrar su experiencia con amigos, visitantes o estudiantes en sus frecuentes instancias de capacitación difundidas por su página web o redes sociales. Articulando su visión comunitaria a una perspectiva económica solidaria. En efecto, uno de sus miembros refiere que se trata de: "una forma de producción justa con los agricultores, trabajadores y compradores" (CP, enero de 2018). Llama la atención las contradicciones sobre el uso del dinero como articulador de la vida comunitaria y su relación con las dinámicas financieras de la sociedad chilena, ante lo cual observamos que las contradicciones son explícitamente abordadas y los conflictos morales que podrían surgir en los participantes, toman una salida pragmática, en la cual puede mediar el dinero $u$ otras formas de moneda social que valore la reciprocidad. Sostienen que su forma de vida se trata de una perspectiva holística que implica el desarrollo de la persona y que debe permitir "aplicar soluciones a los desafíos sistémicos de la vida humana en el siglo 21" (CP, enero de 2018).

\section{Conclusión}

En el transcurso de la historia vemos que la utopía aparece como un impulso de disconformidad con lo que prevalece y una constante búsqueda de bienestar y justicia social. En este contexto, asistimos a la emergencia de iniciativas utópicas en la sociedad contemporánea, las cuales muestran una intensificación de su presencia en distintos lugares del mundo, y que en Chile han adquirido una expresión particularmente importante, en lugares dotados de ecosistemas de alto valor ecológico y en el contexto de una sociedad tensionada por un modelo de desarrollo neoliberal en su versión más ortodoxa. Así, se verifica intensa actividad de comunidades utópicas a lo largo del país. Estas iniciativas representan impulsos creativos que intentan responder con distintos modos de convivencia a las tensiones propias de la sociedad moderna capitalista.

Estas CIU, en muchos casos, están asociadas a una incipiente actividad turística y se nutren de la migración de personas desde las ciudades las cuales buscan reinventarse y desarrollar modos de convivencia distintos a la que comúnmente encontramos en nuestra sociedad. Es frecuente la compra de terrenos para la concreción de su utopía, modificando el lugar de acuerdo con sus 
ideales y resignificándolo en un ejercicio interpretativo entre sus creencias, afectos y las características naturales del entorno (Zunino y Huliñir, 2017). Esto implica que las zonas en donde se instalan son lugares de alta demanda por sujetos interesados en su adquisición como propiedad. Por lo tanto, en muchos casos la utopía se facilita por las condiciones materiales de la vida neoliberal. Instalándose en espacios en donde, además convergen dinámicas socioespaciales de penetración del capitalismo. Consideramos que las zonas de mayor concentración de CIU en el Chile se insertan en un creciente mercado del turismo espiritual en el valle del Elqui (Bahamondes, 2017) y en áreas sujetas a una actividad inmobiliaria relacionada con la presencia de amenidades naturales (Hidalgo, Borsdorf y Plaza, 2009). También observamos una concentración de $\mathrm{CIU}$ en el centro-sur de Chile, en áreas de productividad agrícola, o en un sentido diferente, en la norpatagonia chilena, caracterizada como una reserva de vida abierta a la apropiación mercantil bajo un discurso capitalista verde (Núñez, Aliste y Bello, 2016), o por la atracción de sus paisajes prístinos e inspiradores, abiertos a interpretaciones impredecibles de sujetos agrupados en torno a una utopía (Huliñir y Zunino, 2017).

Mas allá de la efectividad de estas iniciativas comunitarias para transformar la situación social, nos parece importante destacar que sus experiencias cumplen un rol central para mostrar que existen alternativas fuera del orden social actual, en donde los saberes y prácticas terapéuticas, indígenas, espirituales y ecológicas brindan un marco de significados que configuran la cooperación y desarrollo de los participantes. A pesar de que se han reportado incongruencias de estas comunidades al convertirse en jardines privados para el cultivo del ego de un grupo selecto, en un ambiente de fiesta (Zunino y Huiliñir, 2017).

Aunque reconocemos lógicas modernas en los distintos modos de convivencia comunitaria, consideramos que la noción de utopía postmoderna (González, 2010) captura, en parte, elementos que aquí hemos descrito. Consideramos que los sujetos, además de la búsqueda intencional de utopías comunitarias que desafíen la modernidad, también buscan una contención existencial y afectiva en el presente, siendo la comunidad un espacio social romantizado que media y protege las relaciones cotidianas para una gratificación personal y un ejercicio para enfrentar los problemas que afectan a la sociedad. En efecto, pensamos que la utopía comunitaria busca el desarrollo del individuo y orienta una serie de procedimientos para el trabajo o gestión del Self.

Más que un relato único con bordes precisos, en la manifestación empírica de las CIU observamos un traslape de prácticas y significados, que muestran como las tipologías descritas, si bien adecuadas para identificar el sentido general que adquieren las practicas, deben entenderse como una herramienta descriptiva dinámica en permanente diálogo con una realidad compleja y cambiante. Sumado a lo anterior, también destacamos la capacidad de los participantes para pensar e imaginar sus convicciones en su mundo interno. En este sentido, observamos un sujeto percibido como inacabado, en constante transformación, asumiendo y tensionando los valores modernos de elección y creación de su propia identidad personal. Esta situación pone en el núcleo de estas iniciativas a los conocimientos que sustentan la utopía y a la racionalización como estrategia personal para pensar críticamente la sociedad, pero al mismo tiempo para dar espacio a sus desbordes utópicos en situaciones de contradicción. Cabe mencionar que no en todos los casos esta racionalidad parece lineal, material o pragmática, sino que, los participantes se apropian de conocimientos diversos que entregan otras figuras al ejercicio de pensar su propia existencia y la existencia de lo real. Mientras que, por otro lado, reconfiguran la materialidad, o de 
plano la ignoran, y con ello también ignoran las condiciones materiales que permiten la concreción de su utopía. En algunos casos se ignoran las dinámicas de clase social, o se centran en un individualismo optimista o compasivo que aleja al proyecto utópico de la transformación social esperada y que solo se restringe a una experiencia individual o grupal de felicidad, que más bien contribuyen al engrandecimiento del yo (Gebauer et al., 2018).

En síntesis, consideramos que la intensificación de la presencia de CIU en Chile muestra impulsos de reinvención del sujeto moderno y del lugar en el contexto de la modernidad capitalista, en donde el sujeto es llamado a desarrollar su potencial individual para transformar la sociedad, poniendo en práctica procedimientos diversos que finalmente se dirigen al propio sujeto y su (in) dependencia de la sociedad.

\section{Referencias bibliográficas}

ACHOUCH, Y. \& MORVAN, Y. The kibutz and "developmen Towns" in Israel. Zionist Utopias: Ideals ensnared in a tormented history. Justice spatiale-spatial justice, 2012, vol. 1, n 5, p. 1-24.

ARDILA, R. La utopía psicológica: Walden, Walden Dos y Walden. Suma Psicológica, 2004, vol. 11, $n^{\circ} 2$, p. 145-159.

BAHAMONDES, L.; DIESTRE, F.; MARÍN, N. y RIQUELME, W. Espiritualidad y territorio: la emergencia de nuevos mercados religiosos en Pisco Elqui (IV Región, Chile). Revista de Estudios Sociales, $2017, n^{\circ} 61$, p. $69-84$.

BAUMAN, Z. Modernidad y ambivalencia, Barcelona: Anthropos, 2005, 380p.

BERGER, A. The Rainbow Family: an ethnography of spiritual postmodernism. Tesis de Doctorado. University of St Andrews, 2006, 293p.

BERIAIN, J. El sujeto transgresor (y transgredido). Modernidad, religión, utopía y terror. Barcelona: Anthropos, 2011, 189p.

BORZUTZKY, S. From Chicago to Santiago: Neoliberalism and social security privatization in Chile. Governance, 2005, vol. 18, n 4, p. 655-674.

BRÖCKLING, U. El self emprendedor. Sociología de una forma de subjetivación. Santiago de Chile: Ediciones Universidad Albero Hurtado, 2015, 335p.

CANCINO, L. Acción colectiva e imaginarios en contextos de interés medioambiental. Una etnografía a los Asentamientos pro Sustentabilidad en Chile. Investigaciones Geográficas, 2018, p. 45-79. https://doi.org/10.5354/0719-5370.2018.51555

CASTRO-GÓMEZ, S. Historia de la gubernamentalidad I: Razón de Estado, liberalismo y neoliberalismo en Michel Foucault. Bogotá: Siglo del Hombre Editores, 2015, 276p. 
CASTRO-GÓMEZ, S. Tejidos oníricos: Movilidad, capitalismo y biopolítica en Bogotá (19101930). Bogotá: Editorial Pontificia Universidad Javeriana, 2009, 282p.

CENZATTI, M. Heterotopias of difference. En DEHAENE, Michiel y DE CAUTER, Lieven (eds.). Heterotopia and the city. Public space in a postcivil society. Londres/New York: Routledge, 2008, p. 75-86.

CHAVES, M., MACINTYRE, T., VERSCHOOR, G., \& WALS, A. Radical ruralities in practice: Negotiating buen vivir in a Colombian network of sustainability. Journal of Rural Studies, 2018, vol. 59, p. 153-162.

$\mathrm{CHOI}, \mathrm{J} . \mathrm{S}$. Charasteristics of community life in foreing intentional communities focus on the differences between ecovillage and cohousing. International Journal of Human Ecology, 2008, vol. 9, p. 93-105.

CHRISTIAN, D. Finding community: how to join an ecovillage or intentional community. Canadá: New Society Publishers, 2009, 243p.

CLARK, T. D. The Paradox of the Neoliberal Developmentalist State: Reconstructing the Capitalist Elite in Pinochet's Chile. In: North L., Clark T. (eds) Dominant Elites in Latin America. Latin American Political Economy. Palgrave Macmillan, Cham, 2018. p. 23-56.

CONCHA, C. La Ecoaldea El Romero. Etnografía a una comunidad alternativa de nuestro país. Tesis de licenciatura. Universidad de Chile, 2010, 144p.

DE MATHEUS, L. Viver de forma sustentável ou contribuir para a sustentabilidade do capital? As contradições que permeiam a práxis das ecovilas em tempos neoliberais. Revista Geografias, 2014, vol. 10, n 1, p. 41-53.

ECO-YOGA VILLAGES. About us. 2018- Disponible en internet: http://ecoyogavillages.org/en/ about-us/

EKSVÄRD, K. \& RYDBERG, T. Integrating participatory learning and action research and systems ecology: A potential for sustainable agriculture transitions. Systemic Practice and Action Research, 2010, vol. 23, n 6, p. 467-486.

ESCOBAR, P. The new labor market: the effects of the neoliberal experiment in Chile. Latin American Perspectives, 2003, vol. 30, no 5, p. 70-78.

ESCORIHUELA, J. Camino se hace al andar: del individuo moderno a la comunidad sostenible: manual para tansicioneros. Nous Editorial, 2008. 201p.

FELLOWSHIP FOR INTENTIONAL COMMUNITY. Communities Directory: Guide To Cooperative Living (7th edition). Missouri: Rutledge, 2016, 608p. 
FERREIRA, C. Modernidade: rupturas com a tradicao. Diálogos. Revista de estudos culturais e da contemporanidade, 2013, vol. 8, $n^{\circ} 1$, p. 119-134.

FIGUEROA, M. E., \& LÓPEZ, L. Imaginarios y utopías: Un punto de encuentro. Política y Cultura, 2014, vol. 41, p. 169-190. Disponible en http://www.scielo.org.mx/scielo.php?script=sci_arttext\&pid=S0188-77422014000100008\&lng=es\&tlng=es

FOTOPOULOS, T. Is the eco-village movement a solution or part of the problem. The International Journal of Inclusive Democracy, 2006, vol. 2, n³.

FOURIER, C. El nuevo mundo industrial y societario. México: Fondo de la Cultura Económica, 1989, $511 \mathrm{p}$.

GEBAUER, J., NEHRLICH, A. D., STAHLBERG, D., SEDIKIDES, C., HACKENSCHMIDT, D., SCHICK, D., ... \& MANDER, J. Mind-body practices and the self: yoga and meditation do not quiet the ego, but instead boost self-enhancement. Psychological Science, 2018, p. 1-10.

GERGEN, K. El yo saturado: dilemas de identidad en el mundo contemporáneo. Barcelona: Paidós, 2006, 409p.

GLOBAL ECOVILLAGE NETWORK [GEN]. Projects, 2018. Disponible en internet: https://ecovillage. org/projects/

GONZÁLEZ, A. Ir a más allá del descontento de la postmodernidad. Recuperar los aportes críticos de José María Mardones, in memoriam. Xipe Totek: Revista trimestral del Departamento Filosofía y Humanidades ITESO, 2010, vol. 19, nº 74, p. 128-143.

GUBER, R. La etnografía: método, campo y reflexividad. Bogotá: Editorial Norma, 2001, 146p.

HALL, R. The ecovillage experience as an evidence base for national wellbeing strategies. Intellectual Economics, 2015, vol. 9, n¹, p. 30-42.

HARVEY, D. Espacios de esperanza. Madrid: AKAL, 2003, 328p.

HIDALGO, R.; BORSDORF, A. \& PLAZA, F. Parcelas de agrado alrededor de Santiago y Valparaíso: ¿Migración por amenidad a la chilena? Revista de Geografía Norte Grande, 2009, n 44, p. 93-112.

HO, W. S.; HASHIM, H. \& LIM, J. S. Integrated biomass and solar town concept for a smart eco-village in Iskandar Malaysia (IM). Renewable Energy, 2014, vol. 69, p. 190-201.

HONG, S. \& VICDAN, H. Re-imagining the utopian: Transformation of a sustainable lifestyle in ecovillages. Journal of Business Research, 2016, vol. 69, n 1, p. 120-136.

HOOD, B. et al. Decentralised development:the ecovillage at currumbin. Water, 2010, p. 37-43. Disponible en internet: https://research-repository.griffith.edu.au/handle/10072/37211 
HUILIÑIR-CURÍO, V.; ZUNINO, H. Movilidad, utopías y lugares híbridos en Los Andes del sur de Chile. Revista INVI, 2017, vol. 32, nº 91, p. 141-160.

KIM, E. S., PARK, D. K., ZHAO, X., HONG, S. K., KOH, K. S., SUH, M. H., \& KIM, Y. S. Sustainable management of grassland ecosystems for controlling Asian dusts and desertification in Asian continent and a suggestion of Eco-Village study in China. Ecological Research, 2006, vol. 21, n 6, p. 907-911.

KIRBY, A. Redefining social and environmental relations at the ecovillage at Ithaca: A case study. Journal of Environmental Psychology, 2003, vol. 23, n 3, p. 323-332.

LANDER, E. Ciencias sociales: saberes coloniales y eurocéntricos. En: LANDER, Edgardo (ed.), La colonialidad del saber: Eurocentrismo y ciencias sociales. Perspectivas Latinoamericanas. Buenos Aires: CLACSO, 2000, p. 11-40.

LIODAKIS, G. Capital, Economic Growth, and Socio-Ecological Crisis: A Critique of De-Growth. International Critical Thought, 2018, vol. 8, n 1, p. 46-65.

LITFIN, K. Ecovillages: Lessons for Sustainable Community. Cambridge: Polity Press, 2014, 223p.

LOCKYER, J. From Earthships to Strawbales: Sustainable Housing in Ecovillages. Anthropology News, 2008, vol. 49, n 9, p. 20-20.

MARDONES, R.E. Apropiación del espacio público y acción colectiva juvenil: estudio de caso en una ciudad de Chile. Interamerican Journal of Psychology, 2015, vol. 49, no 3.

MARDONES, R.E; ULLOA, J. Construcción subjetiva del territorio: Experiencias del habitar la provincia del Bio Bio, Chile. Estudos de Psicologia, 2017, vol. 22, no 4, p. 422-431.

MARDONES, R.E; ULLOA, J.; SALAS, G. Usos del diseño metodológico cualitativo en artículos de acceso abierto de alto impacto en ciencias sociales. Forum Qualitative Sozialforschung/Forum: Qualitative Social Research, 2018, vol. 19, n¹, p. 18.

MARDONES, R.E; ZUNINO, H. Repensando lo comunitario: discursos de comunidades utópicas en Chile. Convergencia Revista de Ciencias Sociales, 2019, N 81. http://dx.doi.org/10.29101/crcs. v0i81.10615

MATHEWS, F. Beyond modernity and tradition: a third way for development. Ethics and the Environment, 2006, p. 85-113.

MORO, T. Utopía. Buenos Aires: Espasa-Calpe, [1516]1952

NICOLAS-ARTERO, Ch.; VELUT, S. y ALISTE, E. La expansión del capitalismo en el valle de Elqui: viejas reglas y nuevos espacios. Revista Chilena de Antropología, 2018, n³7, p. 197-212.

NOGUÉ, J. El fenómeno neorrural. Agricultura y sociedad, 1988, vol. 47, p. 145-175. 
NÚÑEZ, A.; ALISTE, E. \& BELLO, Á. Patagonia-Aysén, Reserva de Vida: El discurso de la naturaleza como una nueva utopía capitalista (Chile, siglo XXI). In: XIV Coloquio Internacional de Geocrítica. Las utopías y la construcción de la sociedad del futuro. Universidad de Barcelona, 2016. Disponible en internet: http://www.ub.edu/geocrit/xiv_nunezaliste.pdf

PEREIRA, C. Participación y acción colectiva en los movimientos globales de ecoaldeas y permacultura. Revista Latinoamericana de Psicología, 2013, vol. 45, n 3, p. 399-411.

PÉREZ-LUCO, R.; LAGOS, L.; MARDONES, R. y SÁEZ, F. Taxonomía de diseños y muestreo en investigación cualitativa. Un intento de síntesis entre las aproximaciones teórica y emergente. Ámbitos, 2017, n 39, p. 1-11.

QUIROGA, N. Auroville, ensayo de una humanidad superior. Disponible en internet: https://www. auroville.org/system/file_attachments/files/000/003/270/original/yorokobu_humanidad_superior. pdf?1510470266

RAJOVIĆ, G. \& BULATOVIĆ, J. Eco Tourism with Special Review on Eco-Village "Štavna". Scientific Electronic Archives, 2015, vol. 8, p. 56-65.

SALAMANCA, L. y SILVA, D. El movimiento de ecoaldeas como experiencia alternativa de Buen Vivir. Polis. Revista Latinoamericana, 2015, n² 40, p. 209-231.

SARACHO, F.; HERRERA, D. y GONZÁLEZ, F. Espacios negativos: la construcción de espacios utópicos como resistencia política y su territorialización. XIV Coloquio Internacional de Geocrítica: Las utopías y la construcción de la sociedad del futuro, Universidad de Barcelona, 2016. Disponible en internet: http://www.ub.edu/geocrit/xiv-coloquio/SarachoHerreraGonzalez.pdf

SALAMANCA, L. y SILVA, D. El movimiento de ecoaldeas como experiencia alternativa de Buen Vivir. Polis. Revista Latinoamericana, 2015, n² 40, p. 209-231.

SARACHO, F.; HERRERA, D. y GONZÁLEZ, F. Espacios negativos: la construcción de espacios utópicos como resistencia política y su territorialización. XIV Coloquio Internacional de Geocrítica: Las utopías y la construcción de la sociedad del futuro, Universidad de Barcelona, 2016. Disponible en internet: http://www.ub.edu/geocrit/xiv-coloquio/SarachoHerreraGonzalez.pdf

SARGISSON, L. (2007). Strange Places: Estrangement, Utopianism, and Intentional Communities. Utopian Studies, 2007, vol. 18, n³, p. 393-424. http://doi.org/10.2307/20719884

SARGISSON, L. Fool's Gold? Utopianism in the twenty-first century (1st ed.). London: Palgrave Macmillan, 2012.

SARGISSON, L. Sustainability and the intentional community: green intentional communities. In: Liam Leonard, John Barry (ed.) The transition to sustainable living and practice. Bradford: Emerald Group, 2009, p. 171-192. 
SILVESTRI, M. El propósito de vida en la comunidad intencional. La transformación personal para el cambio micro-social. Tesis de doctorado. Universitat Autònoma de Barcelona, 2015, 211p.

SKINNER, B. Walden dos. Hacia una sociedad científicamente construida. Buenos Aires: Ediciones Orbis, $1968,141 \mathrm{p}$.

SPINK, Peter. Replanteando la investigación de campo: relatos y lugares. Athenea Digital. Revista de pensamiento e investigación social, 2005, vol. 1, no 8.

STUBBLEFIELD, Ch. Managing the Planet: The Anthropocene, Good Stewardship, and the Empty Promise of a Solution to Ecological Crisis. Societies, 2018, vol. 8, n² 2, p. 38.

SULLIVAN, E. (Un)Intentional Community: Power and Expert Knowledge in a Sustainable Lifestyle Community. Sociological Inquiry, 2016, vol. 86, n 4, p. 540-562.

TAYLOR, Ch. Fuentes del yo: la construcción de la identidad moderna. Barcelona: Paidós, 2006, $795 \mathrm{p}$.

THOREAU, H. Walden: o mi vida entre bosques y lagunas. Buenos Aires: Espasa-Calpe, 1949.

VERGARA, L.; SÁNCHEZ, C.; ZUNINO, H. Migración por estilo de vida: ¿Creando comunidades diversas y cohesionadas? El caso de Los Riscos, Pucón, Chile. Revista Austral de Ciencias Sociales, 2019, no 36, p. 47-67.

WALKER, L. EcoVillage at Ithaca. Model for Mainstream·Development? Communities, 1996, vol. 91, p. 41-43. Disponible en internet: https://my.vanderbilt.edu/greencities/files/2014/08/Ecovillage-Ithaca1.pdf

ZUNINO, H. Y HUILIÑIR, V. Capitalismo y heterotopía en la Cordillera de Los Andes del sur de Chile. En: NÚÑEZ, Andrés; ALISTE, Enrique; MOLINA, Raúl (Editores). (Las) Otras Geografías en Chile. Perspectivas sociales y enfoques críticos. Santiago de Chile: Ediciones LOM, 2019, p 349-370.

ZUNINO, H.; HIDALGO, R. En busca de la utopía verde: migrantes de amenidad en la comuna de Pucón, IX Región de La Araucanía, Chile. Scripta Nova, Revista Electrónica de Geografía y Ciencias Sociales, 2010, vol. 14, n³31, p. 75.

ZUNINO, H.; HUILIÑIR-CURÍO, V. La construcción de lugares alternos en la Cordillera de Los Andes del Sur de Chile: utopía y disrupción de la modernidad. En: XIV Coloquio Internacional de Geocrítica: Las utopías y la construcción de la sociedad del futuro, Universidad de Barcelona, 2016. Disponible en internet: http://www.ub.edu/geocrit/xiv-coloquio/ZuninoHuilinir.pdf

ZUNINO, H.; HUILIÑIR-CURÍO, V. Utopías modernas y posmodernas en el Sur de Chile: rupturas y continuidades. En SÁNCHEZ, Rafael; HIDALGO, Rodrigo; ARENAS; Federico (comps.). Re-conociento las geografías de América Latina y el Caribe. Santiago de Chile: Pontificia Universidad Católica de Chile, Instituto de Geografía, 2017, p. 157-182. 
